\title{
APPLICABILITY OF VARIOUS INTERPOLATION APPROACHES FOR HIGH RESOLUTION SPATIAL MAPPING OF CLIMATE DATA IN KOREA
}

\author{
Ayeong Jo ${ }^{1}$, Jieun $\mathrm{Ryu}^{2}$, Heyin Chung ${ }^{3}$, Youyoung $\mathrm{Choi}^{3}$, Seongwoo Jeon ${ }^{3, *}$ \\ ${ }^{1}$ Dept. of Lifescience, Korea University, Seoul, Korea - ayoung6432@korea.ac.kr \\ ${ }^{2}$ Environmental GIS/RS Center, Korea University, Seoul, Korea - mail \\ ${ }^{3}$ Dept. of Environmental Science \& Ecological Engineering, Korea University, Seoul, Korea - eepps_korea@korea.ac.kr
}

\section{Commission III, ICWG IV/III}

KEY WORDS: Climate Change, Interpolation, IDW, Cokriging, Kriging, Precipitation, Temperature

\begin{abstract}
:
The purpose of this study is to create a new dataset of spatially interpolated monthly climate data for South Korea at high spatial resolution (approximately 30m) by performing various spatio-statistical interpolation and comparing with forecast LDAPS gridded climate data provided from Korea Meterological Administration (KMA). Automatic Weather System (AWS) and Automated Synoptic Observing System (ASOS) data in 2017 obtained from KMA were included for the spatial mapping of temperature and rainfall; instantaneous temperature and 1-hour accumulated precipitation at 09:00 am on 31th March, 21th June, 23th September, and $24^{\text {th }}$ December. Among observation data, 80 percent of the total point (478) and remaining 120 points were used for interpolations and for quantification, respectively. With the training data and digital elevation model (DEM) with 30m resolution, inverse distance weighting (IDW), co-kriging, and kriging were performed by using ArcGIS10.3.1 software and Python 3.6.4. Bias and root mean square were computed to compare prediction performance quantitatively. When statistical analysis was performed for each cluster using $20 \%$ validation data, co kriging was more suitable for spatialization of instantaneous temperature than other interpolation method. On the other hand, IDW technique was appropriate for spatialization of precipitation.
\end{abstract}

\section{INTRODUCTION}

As the global interest in climate change increases, and the development of computer technology and the construction of high-resolution geographical information accelerate, studies using high-resolution lattice climate data have been actively conducted in various fields such as environmental ecology, agricultural weather, geography, and atmospheric environment (Fick and Hijmans, 2017). High-resolution lattice climate data is used as an important basis for climate modeling, statistical analysis, ecosystem and habitat research and climate spatialization research (Lettenmaire et al., 2008). In addition, the need for lattice climate data is increasing in that it provides scientific and logical information on climate change policy decisions, including climate change impact assessment and adaptation policy.

In order to generate high resolution lattice climate data, a climate map would be constructed via using satellite imagery or interpolation with observation data (Chervenak et al., 2003). Although satellite images with more detailed information can be acquired and applied easily than in the past, there are disadvantages as it is difficult to collect images suitable for the study period, the amount of data to be processed is large, and highly specialized knowledge is required (Ninyerola et al., 2007, Daly, 2006).

When interpolating observational data, mechanical or geostatistical interpolation can be used. Although mechanical interpolation has the advantage of producing physically accurate meteorological data, it has limitations in that it requires high computing power and computation time (Jang et al., 2015).

Statistical interpolation method has the advantage of being able to perform experiments on a general desktop computer and has a short computation time (Park and Jang, 2011), but uncertainty appears in the step of estimating a value in a grid which is not sampled outside the observation point (Jeong and Cho, 2011). Therefore, in order to overcome the problem of uncertainty when using a simple spatial interpolation technique, additional data such as remote sensing data, GIS thematic map, elevation and inclination have been actively applied in the interpolation process (Creutin et al., 1998, Daly et al., 2003). Especially in Korea, considering the high temporal and spatial variability of meteorological factors due to the complex terrain and monsoon (Baek and Jang, 2011), numerical studies have been reported on statistical interpolation using digital elevation model(DEM) or remote sensing data in order to overcome the limitations of uncertainty (Park, 2009, Park, 2008).

In this study, the statistical interpolation method were adopted as it has the easiest access to the study considering the advantages and disadvantages of each method. Using the temperature and precipitation data and DEM, the seasonal temperature and precipitation map of South Korea were constructed.

We applied Inverse Distance Weighting (IDW), kriging, and co-kriging, which are the most representative spatial statistical methods. In order to compare the accuracy of statistical interpolation, the results were compared with the local forecast model (LDAPS, $1.5 \mathrm{~km}$ resolution), which is provided by Korea Meteorological Administration.

\section{RESEARCH MATERIALS}

The materials used in this study are largely composed of lattice climate data of local forecast model, weather observation climate data and DEM.

\footnotetext{
* Corresponding author
} 


\subsection{Local Forecast Model (LDAPS) Lattice Climate Data}

In this study, lattice climatic data of the local forecasting model provided by KMA were used to compare and analyze the applicability of the temperature and precipitation distributions constructed via various spatial interpolation methods. The numerical forecast provided by KMA predicts future weather from the current atmospheric state using a numerical forecasting model calculated by a high-performance computer.

The numerical forecasting model is a meteorological modeling of the Earth's meteorological system using aerodynamic and physical equations that govern atmospheric conditions and motion. In this process, a large amount of numerical operation is required within a limited time depending on the data type, size, and resolution. Therefore, it is necessary to create and execute the program using a special programming environment provided by a supercomputer designed for high performance numerical calculation.

The data of the global, regional, and local forecasting models, three major numerical forecasting models of KMA, are provided with two types of data. The backscattering data is the most widely used numerical forecasting data and expresses the state of the atmosphere in three dimensions. Single-surface data represent the meteorological elements that occur only in a single layer of meteorological elements, such as precipitation and the variables defined in the soil layer.

In this study, the single-surface data for the local forecasting model (LDAPS, Local Data Assimilation and Prediction System), performing 8 times a day by receiving the boundary field from the global model every 3 hours $(00,06,12$, 18UTC: 36 hours Prediction, 03, 09, 15, 18UTC: 3 hour prediction), was used. It is composed of 70 layers up to about $40 \mathrm{~km}$ in vertical direction and has $1.5 \mathrm{~km}$ spatial resolution. In this study, in order to calculate the most recent climate lattice data for temperature and precipitation, instantaneous temperature and 1 hour cumulative precipitation among 78 variables for 2017 were used. In addition, considering the features of local forecast data we used 00UTC (Korean time 09:00) and 00hr data.

Since Korea ordinarily determines the bifurcation point of the seasons as the position of the sun, seasonal comparisons were conducted for the vernal equinox, spring, autumn, and winter, which are determined according to the altitude of the sun during the 24 seasons (Jin and Park, 2015). The equinox are located in the equator. The sun is located on the Tropic of Cancer, and the highest altitude of the Sun is the highest. The comrades are located on the Tropic of Cancer. To best eliminate the meteorological factors such as wind, localized rain trough, that could affect to create a climate grid data, March 31, June 21, September 23, December 24 were chosen as the subject of study. The spatial mapping of instantaneous temperature has been carried out by considering the effects of wind, clouds and humidity. Each day was selected according to the quantitative criteria; average wind speeds of less than $2 \mathrm{~m} / \mathrm{s}$, average cloud volume less than 3.5 and average relative humidity of less than $80 \%$. For spatial mapping of the mean cumulative precipitation over 1 hour, except for the local rainy days of March and December, the nearest day to the equinoxes and comrades was adopted, which rained national wide due to the effects of cyclone passing through Korea.

\subsection{Meteorological Observed DATA and DEM}

In this study, spatial interpolation was performed using Automated Surface Observing System (ASOS) and automated weather system (AWS) as terrestrial observation data provided by KMA. A total of four instantaneous temperatures and two 1hr accumulated rainfall lattice data were constructed with the time observation data from 595 observations (480 AWS, 95 ASOS) in the study area, measured on March 21, June 21, September 23, and December 24, 2017 at 09:00 (Figure 1. (a)). For lattice climatic data production, about $80 \%$ of 595 terrestrial observation data (478) were used, and the remaining 117 data were used for verification (Table 1.).

\begin{tabular}{|c|c|c|c|c|c|}
\hline & 03.31 & 06.21 & 09.23 & 12.24 \\
\hline $\begin{array}{c}\text { Temp } \\
\left({ }^{\circ} \mathrm{C}\right)\end{array}$ & Max & -4.3 & 13.3 & 11 & -4.4 \\
& Mean & 7.8 & 23.2 & 19 & 4.9 \\
\hline \multirow{3}{*}{$\begin{array}{c}\text { Prep } \\
(\mathrm{mm})\end{array}$} & Min & 0 & NA & NA & 0 \\
& Mean & 7.1 & NA & NA & 20.7 \\
\hline
\end{tabular}

Table 1. Observed data of instantaneous temperature and precipitation in 2017.

Geographical environmental variables related to temperature and precipitation characteristics generally include elevation, distance from shoreline, land cover, and slope orientation. Among these, the elevation has the greatest effect on the variability of climate factors, with the temperature dropping by $0.6^{\circ} \mathrm{C}$ as altitude increases by $100 \mathrm{~m}$ in the troposphere (Baek and Jang, 2011). Therefore, the top factor influencing the temporal and spatial variation of climate factors is the elevation above sea level. The DEM data used in the study is the Shuttle Radar Topography Mission (SRTM) DEM provided by EarthExplorer and the spatial resolution is $30 \mathrm{~m}$ (1 arc second). (Figure 1(b))
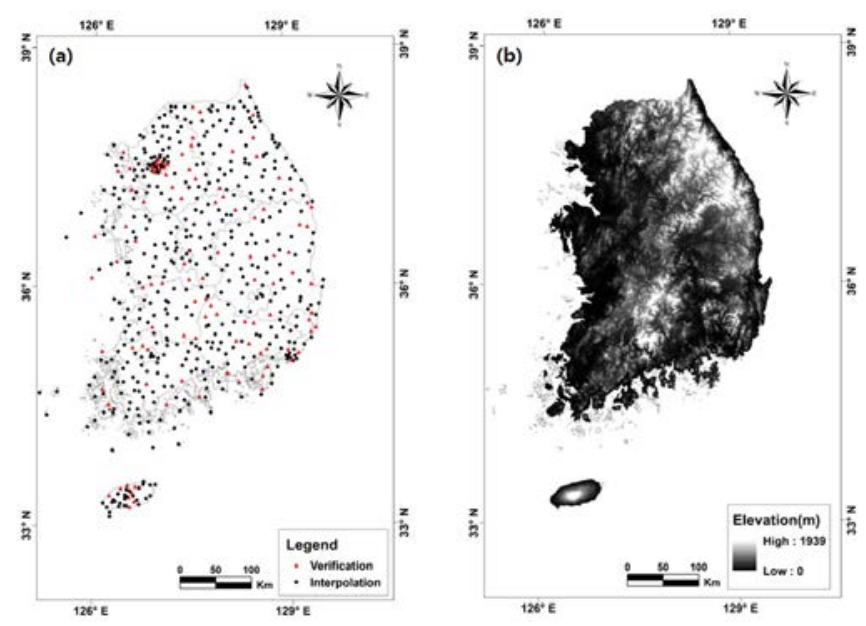

Figure 1. (a) Location of AWS and (b) DEM of study area.

\section{RESEARCH METHOD}

\subsection{Cluster Analysis}

South Korea, the subject of this study, is characterized by the large climate difference between south and north and east and west due to the long north-south direction and the mountains developed from north to south (Park, 2014). Due to these topographical factors, various studies have been carried out on the features of the temperature and precipitation of Korea considering the effects of altitude and slope. 


\begin{tabular}{|c|c|c|c|}
\hline & Cluster1 & Clsuter2 & Cluster3 \\
\hline & & & \\
Spatial & & & \\
distribution & $\ddots$ & & \\
& & & \\
& & & \\
\hline Feature & High & Middle & Low \\
Slope ${ }^{\circ}$ ) & 21.4 & 13.5 & 5.1 \\
Elevation(m) & 942 & 387 & 60 \\
Number & 24 & 98 & 473 \\
Training & 20 & 79 & 379 \\
Test & 4 & 19 & 94 \\
\hline
\end{tabular}

Table 2. Cluster analysis with elevation and slope

In this study, hierarchical cluster analysis of observation data was performed to analyze the differences of result according to altitude and slope (Table 2.). Ward connection method, one of the hierarchical analysis used in this study, is to grouping together the data in each stage of cluster analysis, by measuring the error sum of squares between the mean and the data of the cluster thereby to merge so as to minimize its error sum value (Punj and Stewart, 1983).

As a result of clustering of observation data with altitude and slope as variables, a total of three significant clusters were derived. After that, K-average algorithm was used to set the cluster-specific labels, and $80 \%$ training set for interpolation and $20 \%$ test set for validation were randomly selected in each cluster.

\subsection{Statistical Interpolation}

\subsubsection{Inverse Distance Weighting (IDW)}

One of the interpolation methods, inverse distance weighting (IDW), is a technique that reflects the influence of observations as the closer the distances of known values are located when estimating the values for unobserved points. That is, it is based on the assumption that the weight decreases exponentially as the distance between the unobserved point and the neighboring observation point increases. The basic formula for IDW is:

$z_{g}(x)=\sum_{i=1}^{N} \alpha_{i} Z\left(x_{i}\right)$

where $\quad z_{0}(x)=$ the predicted value at position $x$

$N=$ weight for observation point $x_{1}$

$Z\left(x_{i}\right)=$ observation value at observation point $x_{1}$

$\alpha_{i}=$ weight

Since the weight is inversely proportional to the distance, its value decreases as the distance increases. Finally, it can be expressed by the following equation:

$\alpha_{i}=\frac{\left(1 / d_{i}\right)^{P}}{\sum_{i=1}^{N}\left(1 / d_{i}\right)^{P}}$ where $\quad \begin{aligned} & d_{1}=\text { distance between } x_{1} \text { and } x \\ & P=\text { degree of change in weight according to distance }\end{aligned}$

If $P$ is 0 , the result of IDW is equal to the arithmetic mean, and when it is increased to infinity, it becomes equal to the estimated result of the Thiessen method (Hwang and Ham, 2013).

\subsubsection{Kriging}

One of the commonly used spatial interpolation methods is to derive an unknown value as a linear combination of random variables with spatial correlation. By considering the correlation between the distance from the measured value and the neighboring values, it statistically measures the variability of the distance between the measured values. And then, based on the variogram, it performs interpolation. Uni-variate kriging includes simple kriging, ordinary kriging, universal kriging, and block kriging.

In this study, ordinary kriging was used to minimize the variance of error. Ordinary kriging assumes that the mean is constant locally based on the estimated point. Its estimates at any lattice point is expressed:

$$
\begin{aligned}
Z_{g}(x) & =E(x)+\sum_{i=1}^{N(x)} \alpha_{i}(x)\left[Z\left(x_{i}\right)-E\left(x_{i}\right)\right] \\
& =\sum_{i=1}^{N(x)} \alpha_{i}(x) Z\left(x_{i}\right)+\left[1-\sum_{i=1}^{N(x)} \alpha_{i}(x)\right] E(x)
\end{aligned}
$$

If the sum of the kriging weights is equal to 1 , then the local mean value can be eliminated and the normal kriging estimate is:

$$
Z_{g}(x)=\sum_{i=1}^{N(x)} \alpha_{i}(x) Z\left(x_{i}\right), \sum_{i=1}^{N(x)} \alpha_{i}(x)=1
$$

Here, the ordinary kriging weights can be estimated by minimizing the estimated variance (Hwang and Ham, 2013).

\subsubsection{Co-Kriging}

Co-kriging is a method designed to compensate for the disadvantages of uni-variate kriging, which can improve predictability at unsampled points through multivariate kriging when additional data are available, along with the data under consideration. Multivariate kriging techniques include cokriging, kriging with an external drift (KED), and simple kriging with varing local means (SLKM).

Uni-variate kriging performs interpolation using autocorrelation of the same variable with only one variable value, while co-kriging interpolates by linear combination of two or more variables. In co-kriging, a variable to be predicted is called a peripheral variable, and non-peripheral variables are called a secondary variables.

$$
Z^{*}=\sum_{i=1}^{N(x)} \alpha_{i} z_{i}+\sum_{i=1}^{N_{s}} \sum_{k=1}^{M_{i}} \alpha_{j k} u_{j}\left(x_{j k}\right)
$$

In this case,

$z=$ the number of peripheral variable

$N=$ the total number of the used peripheral variable

$N_{s}=$ the total number of used secondary variables

$u_{i}=j_{\text {th }}$ secondary variables 
The International Archives of the Photogrammetry, Remote Sensing and Spatial Information Sciences, Volume XLII-3, 2018 ISPRS TC III Mid-term Symposium “Developments, Technologies and Applications in Remote Sensing”, 7-10 May, Beijing, China

$$
\begin{aligned}
& m_{1}=\text { total number of } \dot{j} \text { th secondary variables } \\
& \alpha=\text { weight } \\
& \alpha=\text { the position of each data }
\end{aligned}
$$

The total number of $\left(N_{s}+1\right)$ variables and $\left(N+N_{s} \times m_{i}\right)$ data are used to estimate the surrounding values (Jang et al., 2015).

\subsection{Verification}

The bias and mean square error (RMSE) between the instantaneous temperature, 1-hr cumulative precipitation values of the lattice data and 120 validation points corresponding to $20 \%$ were used to verify the distributions generated by each spatial interpolation method. In particular, to verify the interpolation accuracy in each cluster, the bias and RMSE of the spatial interpolation results and LDPAS gridded data from KMA in each cluster were statistically compared.

The mean absolute error (MAE), commonly used for deviation measurements, is an estimate of the uncertainty of the estimated value as the average of the absolute values of the difference between the estimated and actual values. The root mean square error (RMSE), a measure often used to express precision, is the square root of the arithmetic mean of the square sum of the difference (residual) between the estimated value and the actual value (Walther and Moore, 2005);

$\operatorname{Bias}(\mathrm{MAE})$

$$
\frac{\frac{1}{n} \sum_{i=1}^{n}\left|E_{i}-O_{i}\right|}{\sqrt{\frac{1}{n} \sum_{i=1}^{n}\left(E_{i}-O_{i}\right)^{2}}}
$$

where

$$
\begin{aligned}
& \mathrm{E}=\text { estimates } \\
& \mathrm{O}=\text { observations } \\
& \mathrm{n}=\text { total number of samples }
\end{aligned}
$$

In addition, the similarity of spatial pattern between the results of the geo-statistical interpolation and the weather forecasting model lattice climatic data of KMA was analyzed by comparing the spatial patterns of each watershed.

\section{CONCLUSION AND DISCUSSIONS}

In the study of the instantaneous temperature distribution features on March 21th, June 21th, September 23th, and December 24th in 2017, IDW, kriging and co-kriging were applied seasonally to produce a total of 12 lattice temperature data (Table 3., Figure 2.).

As a result, the range between the minimum and the maximum value of the instantaneous temperature derived from statistical interpolation decreased somewhat than the observation data. The mean values were almost similar to the observed data. The decrease in the range between the minimum and maximum values indicates that the spatial continuity of estimates at the verification points have been increased due to the statistical spatial interpolation.

\begin{tabular}{|c|c|c|c|c|c|}
\hline \multicolumn{2}{|c|}{ Temperature $\left({ }^{\circ} \mathrm{C}\right)$} & 03.31 & 06.21 & 09.23 & 12.24 \\
\hline \multirow{2}{*}{ Obs } & Max-Min & 13.2 & 11.6 & 11.9 & 16.7 \\
& Mean & 7.9 & 23.2 & 19.6 & 4.4 \\
\hline \multirow{2}{*}{ CKG } & Max-Min & 8.0 & 8.7 & 8.2 & 13.0 \\
& Mean & 7.8 & 23.1 & 19.7 & 4.4 \\
\hline \multirow{2}{*}{ IDW } & Max-Min & 8.9 & 10.1 & 8.0 & 12.2 \\
\cline { 2 - 6 } & Mean & 7.9 & 23.1 & 19.6 & 4.4 \\
\hline \multirow{2}{*}{ KG } & Max-Min & 6.6 & 6.4 & 6.5 & 12.8 \\
\cline { 2 - 6 } & Mean & 7.8 & 23.2 & 19.7 & 4.5 \\
\hline
\end{tabular}

Table 3. Comparison of temperature estimated by interpolation and observed in 2017. Obs: Observed, CKG: co-kriging, KG: kriging

In order to statistically analyze the results of each interpolation technique, bias and RMSE between the observation points of the verification points were derived (Table 4.). As all of the

\begin{tabular}{|c|c|c|c|c|c|c|}
\hline \multicolumn{3}{|c|}{ Temperature $\left({ }^{\circ} \mathrm{C}\right)$} & 03.31 & 06.21 & 09.23 & 12.24 \\
\hline \multirow{8}{*}{$\mathrm{C} 1$} & \multirow{2}{*}{ CKG } & Bias & 4.62 & 3.03 & 2.58 & 1.74 \\
\hline & & RMSE & 5.03 & 3.58 & 3.26 & 2.37 \\
\hline & \multirow{2}{*}{ IDW } & Bias & 4.18 & 3.09 & 2.34 & 1.72 \\
\hline & & RMSE & 4.79 & 3.49 & 3.01 & 2.35 \\
\hline & \multirow{2}{*}{ KG } & Bias & 5.71 & 3.98 & 2.65 & 1.72 \\
\hline & & RMSE & 5.96 & 4.38 & 3.29 & 2.32 \\
\hline & \multirow{2}{*}{ F } & Bias & 3.73 & 2.05 & 2.73 & 1.03 \\
\hline & & RMSE & 3.86 & 2.48 & 2.83 & 1.67 \\
\hline \multirow{8}{*}{ C2 } & \multirow{2}{*}{ CKG } & Bias & 0.91 & 1.27 & 1.29 & 1.10 \\
\hline & & RMSE & 1.21 & 1.66 & 1.69 & 1.34 \\
\hline & \multirow{2}{*}{ IDW } & Bias & 0.97 & 1.23 & 1.15 & 1.03 \\
\hline & & RMSE & 1.27 & 1.63 & 1.57 & 1.29 \\
\hline & \multirow{2}{*}{ KG } & Bias & 1.13 & 1.23 & 1.22 & 1.12 \\
\hline & & RMSE & 1.43 & 1.64 & 1.59 & 1.39 \\
\hline & \multirow{2}{*}{ F } & Bias & 0.96 & 1.33 & 2.65 & 1.11 \\
\hline & & RMSE & 0.74 & 1.74 & 2.91 & 1.44 \\
\hline \multirow{8}{*}{ C3 } & \multirow{2}{*}{ CKG } & Bias & 0.62 & 0.85 & 0.92 & 0.87 \\
\hline & & RMSE & 0.83 & 1.37 & 1.62 & 1.30 \\
\hline & \multirow{2}{*}{ IDW } & Bias & 0.69 & 0.82 & 1.15 & 1.04 \\
\hline & & RMSE & 1.04 & 1.09 & 2.33 & 1.44 \\
\hline & \multirow{2}{*}{ KG } & Bias & 0.71 & 0.81 & 1.09 & 0.97 \\
\hline & & RMSE & 0.98 & 1.04 & 2.30 & 1.36 \\
\hline & \multirow{2}{*}{$\mathrm{F}$} & Bias & 0.79 & 1.34 & 2.58 & 1.12 \\
\hline & & RMSE & 1.12 & 1.61 & 2.87 & 1.43 \\
\hline
\end{tabular}
interpolation methods showed good results, no significant difference was observed between them. Therefore, bias and RMSE of each cluster were derived with the estimates from statistical spatial interpolation and LDAPS gridded data, and the observation values. When comparing the deviation of each cluster, it can be seen that as the altitude and the slope decreases, the deviation value becomes smaller.

Table 4. Cluster classification and comparision of temperature estimated by interpolation and observed in 2017. C1: cluster1, C2: cluster2, C3: clsuter3, CKG: co-rigging, KG: kriging, F: LDAPS forecast gridded data 

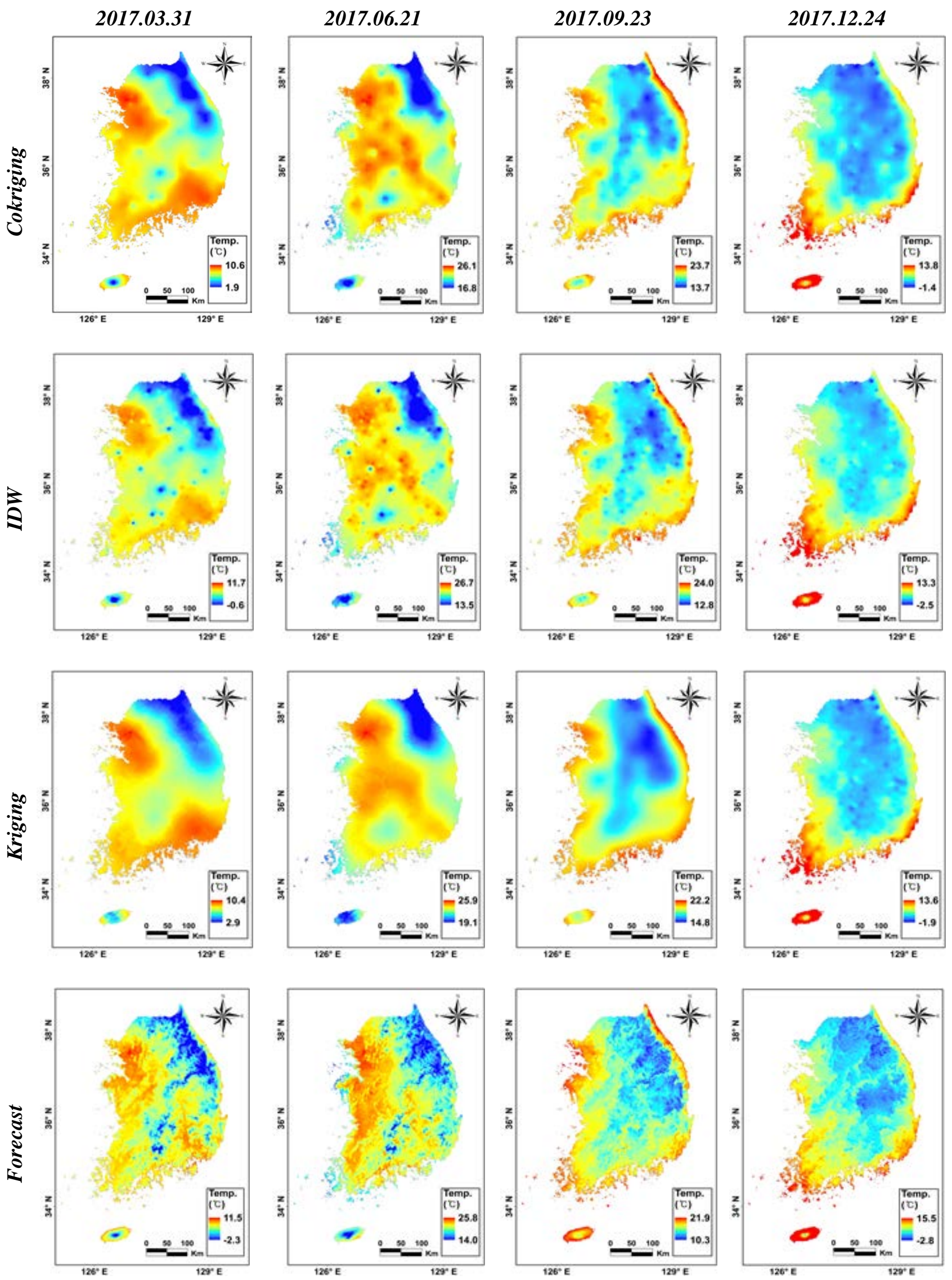

Figure 2. Lattice temperature data constructed via Co-kriging, IDW, Kriging and LDAPS gridded data (Forecast) 
Accuracy of lattice temperature data by interpolation method varied according to the season; high-altitude cluster had higher accuracy on winter season than other seasons, but low-altitude clusters had higher accuracy in spring than winter. The summers and autumn seasons were over-predicted overall over spring and winter seasons. As a result, it can be concluded that the bias of the results via co-kriging and IDW interpolation methods are smaller than kriging, which indicates it is advantageous to calculate lattice temperature data.

As with the bias, overall RMSE value from the results of IDW and co-kriging were smaller than that of kriging, which indicates that IDW and cokriging were useful for constructing lattice temperature data.

When the results of co-kriging and IDW were statistically verified, both bias and RMSE were small, making comparison somewhat difficult. However, when the spatial patterns of the large watershed were compared based on the LDAPS lattice climatic data, it showed that co-kriging reflects local spatial distribution characteristics better than IDW.

In particular, in the case of the East Sea (Han River East Sea and Nakdong River East Sea), the temperature increases rapidly as the altitude decreases with the mountain range. Kriging did not reflect this pattern change, and the distribution pattern of IDW showed a very rapid change, which does not reflect a realistic climate patterns. It can be concluded that the cokriging is able to estimate the spatial distribution by using altitude as an additional variable, which derives more accurate results.

Next, each lattice data for 1-hr cumulative precipitation at 09:00 on March 31th, and December 24th, in 2017 were produced (Figure 3., Table 5.). First, the ranges between the minimum and maximum values were somewhat smaller than that of the observed data, similar to the results of lattice climate data on temperature. This aspect well describes the feature of statistical interpolation which spatial continuity of the estimate at the verification point increases as a result of.

\begin{tabular}{|c|c|c|c|}
\hline \multicolumn{2}{|c|}{ Temperature $\left({ }^{\circ} \mathrm{C}\right)$} & 03.31 & 12.24 \\
\hline \multirow{2}{*}{ Obs } & Max-Min & 4.74 & 15.88 \\
\cline { 2 - 4 } & Mean & 0.65 & 1.97 \\
\hline \multirow{2}{*}{ CKG } & Max-Min & 3.65 & 10.47 \\
\cline { 2 - 4 } & Mean & 0.74 & 2.02 \\
\hline \multirow{2}{*}{ IDW } & Max-Min & 3.86 & 9.81 \\
\cline { 2 - 4 } & Mean & 0.73 & 2.04 \\
\hline \multirow{2}{*}{ KG } & Max-Min & 3.14 & 9.19 \\
\cline { 2 - 4 } & Mean & 0.71 & 1.95 \\
\hline
\end{tabular}

Table 5. Comparision of precipitation estimated by Interpolation and observed in 2017. Obs: Observed, CKG: cokriging, KG: kriging

Similarly to the pattern of temperature, bias and RMSE of precipitation became smaller as the altitude decreased. However, in December 2017, the bias and RMSE at low altitudes were showed the highest value due to the high rainfall in the southwestern plains (Table 6.). Comparing the deviation of precipitation by each interpolation method, IDW showed smaller results than that of kriging or co-kriging.

In March, there was a significant amount of rainfall in eastern Jeju Island, south of the armistice line, and along the Sobaek mountain vein. In December, on the other hand, precipitation

was clearly distinguished near Jeju Island, Nonsan and Honam Plain.

2017.03.31
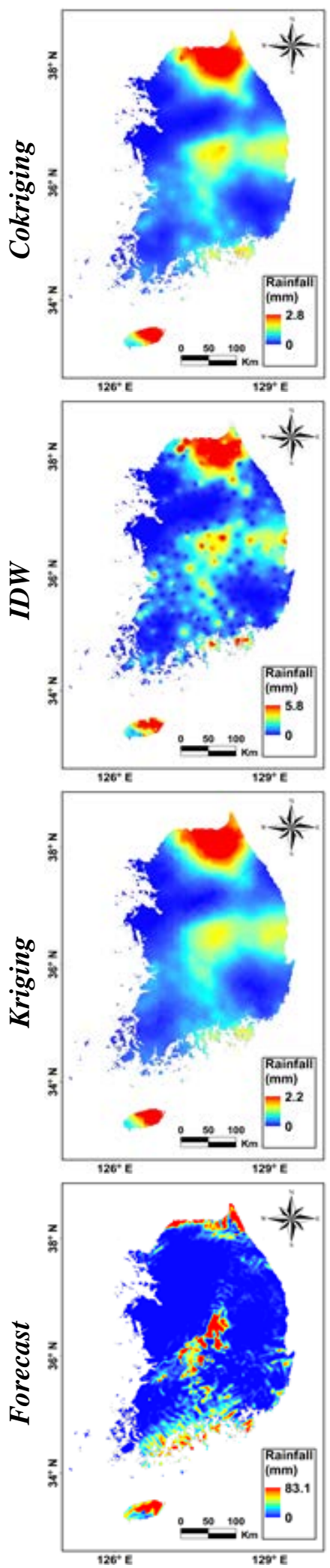

Figure 3. Lattice 1-hr cumulative precipitation data constructed via Co-kriging, IDW, Kriging and LDAPS gridded data (forecast)
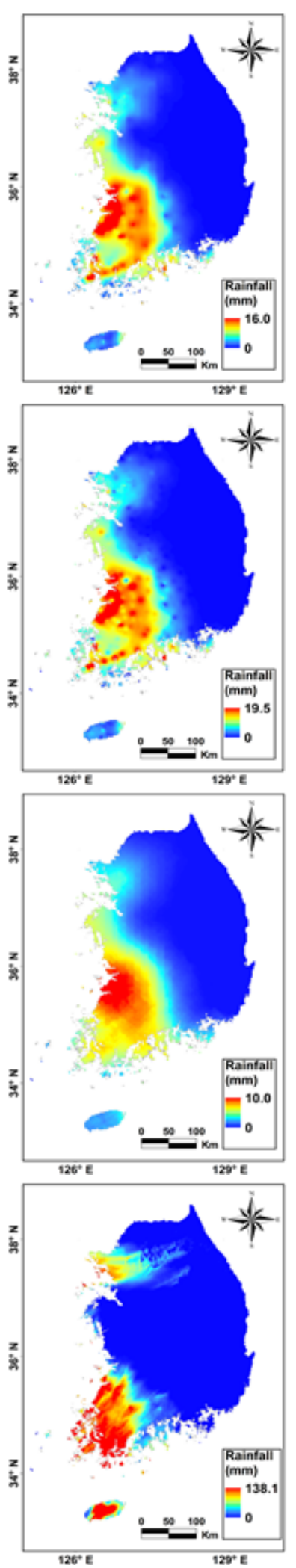
. (1) 
In the case of precipitation, unlike the temperature, the results of IDW well described the characteristics of precipitation, which shows a rapid change and small regional continuity, rather than the other two interpolation methods. Co-kriging and kriging did not show any significant differences in distribution pattern and statistical test results.

Considering the statistical test result and the precipitation distribution pattern of each interpolation method, it is considered that other topographical and climatic factors such as distance from altitude coastline, slope direction, and trough of low pressure would affect the rainfall distribution pattern.

\begin{tabular}{|c|c|c|c|c|}
\hline \multicolumn{3}{|c|}{ Temperature $\left({ }^{\circ} \mathrm{C}\right)$} & 03.31 & 12.24 \\
\hline \multirow{8}{*}{ C1 } & \multirow{2}{*}{ CKG } & Bias & 0.50 & 0.83 \\
\hline & & RMSE & 0.63 & 1.54 \\
\hline & \multirow{2}{*}{ IDW } & Bias & 0.36 & 0.72 \\
\hline & & RMSE & 0.45 & 1.40 \\
\hline & \multirow{2}{*}{ KG } & Bias & 0.48 & 0.82 \\
\hline & & RMSE & 0.66 & 1.61 \\
\hline & \multirow{2}{*}{ F } & Bias & 0.83 & 0.75 \\
\hline & & RMSE & 1.15 & 1.53 \\
\hline \multirow{8}{*}{ C2 } & \multirow{2}{*}{ CKG } & Bias & 0.41 & 0.50 \\
\hline & & RMSE & 0.50 & 1.10 \\
\hline & \multirow{2}{*}{ IDW } & Bias & 0.32 & 0.54 \\
\hline & & RMSE & 0.41 & 1.18 \\
\hline & \multirow{2}{*}{ KG } & Bias & 0.43 & 0.61 \\
\hline & & RMSE & 0.53 & 1.39 \\
\hline & \multirow{2}{*}{$\mathrm{F}$} & Bias & 0.82 & 1.58 \\
\hline & & RMSE & 1.08 & 3.26 \\
\hline \multirow{8}{*}{ C3 } & \multirow{2}{*}{ CKG } & Bias & 0.33 & 1.03 \\
\hline & & RMSE & 0.58 & 1.90 \\
\hline & \multirow{2}{*}{ IDW } & Bias & 0.27 & 1.06 \\
\hline & & RMSE & 0.55 & 1.87 \\
\hline & \multirow{2}{*}{ KG } & Bias & 0.33 & 1.04 \\
\hline & & RMSE & 0.59 & 1.83 \\
\hline & \multirow{2}{*}{$\mathrm{F}$} & Bias & 0.28 & 1.65 \\
\hline & & RMSE & 0.76 & 3.15 \\
\hline
\end{tabular}

precipitation estimated by Interpolation and observed in 2017. C1: cluster1, C2: cluster2, C3: clsuter3, CKG: co-kriging, KG: kriging, F: LDAPS forecast gridded data.

This study proposed a method for constructing the gridded climate data in South Korea by comparing the results of statistical interpolation and mechanical interpolation. However, due to the characteristics of the LDAPS model data, there is a limitation in that only the spatialization of the instantaneous temperature and the 1-hr cumulative precipitation has proceeded.

Therefore, future studies will be conducted to construct and compare the lattice climatic data with the gridded climate data from WorldClim in the same time range. In addition, based on the lattice climatic data and WorldClim data, South Korea's bio-climate region will be constructed and compared, in order to evaluate the applicability of the map.

\section{ACKNOWLEDGEMENTS}

This study was carried out with the support of Korea Meteorological Administration as part of Research \& Development Program with a See-At (See+Atmosphere) grant (KMIPA 2015-6140).

\section{REFERENCES}

Seung Gyun,B., Dong Ho, J., 2011, Evalution for Applicability of Cokriging for High Resolution Spatial Mapping of Temperature and Rainfall, Climate Research, 6(3), pp. 242-253.

Stephen, E.F. and Robert J.H., 2017, WorldClim2: new 1-km spatial resolution climate surfaces for global land areas, Int. $J$. Climatol., 37, pp. 4032-4315.

Dongho, J., Nunsol W., Nowook P., 2015, High-resolution Spatial Mapping and Evaluation of Temperature and Rainfall in South Korea using a Simple Kriging with Local Means, Climate Research, 10(2), pp. 165-182.

Yong Seok, K., Kyo Moon, S., Myung Pyo, J., In Tae, C., 2014, Accuracy Comparison of Air Temperature Estimation using Spatial Interpolation Methods according to Application of Temperature Lapse Rate Effect, The Korean Society of Climate Change Research, 5(4), pp. 323-329.

Shepard, D., 1968, A two-dimensional interpolation function for irregularly-spaced data, Proceedings of the $196823^{\text {rd }}$ ACM National Conference, New York, pp. 517-524.

Krige, D.G., 1951, A statistical approach to some mine valuations and allied problems at the Witwatersrand, Master's thesis of the University of Witwaterstand

Lyche, T. and Shchumaker,L.L., 1972, On the convergence of cubic interpolating splines, University of Texas at Austin Center for Numerical Analysis, 61, pp. 1-31.

Creutin, J. D., Delrieu, G., Lebel, T., 2003, Rain measurement by raingage-radar combination: a geostatistical approach, Journal of Atmoispheric and Pceanic Technologies, 5, pp. 102115.

Daly ,C., Helmer, E. H., Maya, Q., 2003, Mapping the Climate of Puerto Rico, Vieques and Culebra, International journal of climatology, 23, pp. 1359-1381.

Jong Chul, P., Man Kyu, K., 2009, A Study on the Use of a Terrain Aspect Variable in Producing the Precipitation Distribution Map applying Cokriging: A Case of Jeju Island, Journal of the Korean Geomorphological Association, 16(3), pp. 59-66.

Nowook, P., Dongho, J., 2008, Mapping of Temperature and Rainfall Using DEM and Multivariate Kriging, The Korean Geographical Society, 43(6), pp. 1002-1015.

Nowook, P., Dongho, J., 2011, Use of Space-time Autocorrelation Information in Time-series Temperature Mapping, The Korean Association of Regional Geographers, 17(4), pp. 432-442. 
Mi Jeong, J., Sun Yurp, P., 2015, Temperature Changes of Climatic Solar Temrs and Their Spatiotemporal Characteristics in South Korea, The Korean Geographical Society, 50(1), pp. 23-36.

Gyoung-Hye, B., Moun-Gjin, L., Byung-Jin, K., 2011, Development of Spatial Statistical Downscaling Method for KMA-RCM by Using GIS, The Korean Association of Geographic Information Studies, 14(3), pp. 136-149.

Seok-Hwan, H., Dae Heon, H., 2013, Evaluation of Spatial Downscaling Methods for Enhancement of Spatial Precipitation Estimation, Journal of KOSHAN, 13(4), pp. 149163.

Bruno, A.W., Joslin, L.M., 2005, The concepts of bias, precision and accuracy, and their use in testing the performance of species richness estimators, with a literature review of estimator performance, Ecography, 28, pp. 815-829.

Soo Jin, P., 2014, Generality and Specificity of Landforms of the Korean Peninsula, and Its Sustainability, The Korean Geographical Society, 49(5), pp. 656-674.

Girish, P., David, W.S., 1983, Cluster Analtsis in Marketing Research: Review and Suggestions for Application, Journal of Marketing Research, 20(2) pp.134-148.

Miquel, N., Xavier, P., Joan, M.R., 2007, Objective air temperature mapping for the Iberian Peninsula using spatial interpolation and GIS, Int. J. Climatol., 27, pp. 1231-1242.

Jongpil, K., Wooseop, L., Hyungon, C., Gwangseob, K., 2014, Estimation of High Resolution Daily Precipitation Using a Modified PRISM Model, Korean Society of Civil Engineers, 34(4), pp. 1139-1150.

Ann, C., Ewa, D., Carl, K., Bill, A., Ian, F., Veronika, N., Jason, L., Alex, S., Arie, S., Bob, D., Dean. W., Don, M., 2003, High-performance remote access to climate simulation data: a challenge problem for data grid technologies, Parallel Computing, 29, pp. 1335-1356.

Christophe,r D., 2006, Guidelines for assessing the suitability of spatial climate data sets, Int. J. climatol., 26, pp. 707-721.

Jaejoon, J., Youngeun, C., 2011, Study on Interpolation Methods to Generate GIS-based Climate Maps, Climate Research, 6(2), pp. 159-170. 